Sir,

\section{Reply to Grzybowski and Ascaso}

We thank Drs Grzybowski and Ascaso ${ }^{1}$ for their interest in and comments on our recent article. ${ }^{2}$ We agree that in our paper there is a lack of details concerning the statistical tests used (which were omitted for the sake of brevity). In the study, we proved that each variable group was normally distributed using the KolmogorovSmirnov normality test. Then we applied the ANOVA for repeated measures test, which best fitted for our analysis. Please note, the Kruskal-Wallis test is valuable only for a two-group comparison. We also agree that the lack of a control group is crucial, and this was acknowledged as a limitation of our study. We also acknowledged the unmasked design of the study as a limitation of our analysis. Regarding the evaluation of posterior hyaloid peeling, our method to describe the intrasurgical findings has been already published by Azzolini et $a l^{3}$ in a study investigating autologous plasmin enzyme for diabetic macular oedema, and, to our knowledge, no other classification systems are available in the literature.

In the conclusion section, we stated that a single intravitreal autologous plasmin enzyme injection seemed to be insufficient to induce a complete posterior vitreous detachment in patients affected by focal vitreomacular traction syndrome, as in our case series, we did not obtain any complete posterior vitreous detachment with a single injection. We thank the authors for the opportunity to clarify this important aspect, which we do not find contradictory. As per our ethical committee approved protocol (reported in the Methods section), we were allowed to perform just one single intravitreal injection for each study patient, with a 24-hour waiting time before vitrectomy. Although we could not ascertain if a greater time gap could have influenced the rate of posterior vitreous detachment occurrence, we remarked that the single injection appeared as a useful tool in vitreoretinal surgery by obtaining an easier-to-peel posterior hyaloid.

Finally, during the revision process of our paper, we preferred to exclude the comparison of our results with the MIVI-IIT study, ${ }^{4}$ as the MIVI-IIT study has a very different study design and uses a different drug. Particularly, we believe that our impossibility (per protocol) to re-inject patients preclude any comparison between the two studies.

\section{Conflict of interest}

The authors declare no conflict of interest.

\section{References}

1 Grzybowski A, Ascaso FJ. Vitreomacular traction syndrome: the role of intravitreal plasmin injection is still not clear. Eye 2013; 27(6): 776.

2 Codenotti M, Maestranzi G, De Benedetto U, Querques G, Della Valle P, Iuliano L et al. Vitreomacular traction syndrome: a comparison of treatment with intravitreal plasmin enzyme vs spontaneous vitreous separation without treatment. Eye 2013; 27: 22-27.
3 Azzolini C, D'Angelo A, Maestranzi G, Codenotti M, Della Valle P, Prati $\mathrm{M}$ et al. Intrasurgical plasmin enzyme in diabetic macular edema. Am J Ophthalmol 2004; 138: 560-566.

4 Stalmans P, Delaey C, de Smet MD, van Dijkman E, Pakola S. Intravitreal injection of microplasmin for treatment of vitreomacular adhesion: results of a prospective, randomized, sham-controlled phase II trial (the MIVI-IIT trial). Retina 2010; 30: 1122-1127.

G Querques, M Codenotti, G Maestranzi, L Iuliano and $\mathrm{F}$ Bandello

Department of Ophthalmology, Vita-Salute University, San Raffaele Scientific Institute, Milan, Italy E-mail: giuseppe.querques@hotmail.it

Eye (2013) 27, 777; doi:10.1038/eye.2013.63; published online 12 April 2013

Sir Comment on 'Vitreomacular traction syndrome: a comparison of treatment with intravitreal plasmin enzyme $v$ s spontaneous vitreous separation without treatment ${ }^{\prime}$

In some patients, incomplete posterior vitreous detachment leads to symptomatic vitreomacular adhesion or vitreomacular traction syndrome. This is a medico-surgical problem in which new therapy is interesting due to the potential prognosis of the untreated disease and its only actual therapy being surgery. Several studies ${ }^{1,2}$, have reported the use of intravitreal proteases such as plasmin, which is able to degrade biochemical glue composed of proteoglycans, including laminin and fibrinectin. The microplasmin is a truncated derivative of plasmin. The product thus obtained has a significantly reduced size and maintains native proteolytic activity. Stalmans et al. ${ }^{1}$ report that intravitreal injection of microplasmin was superior to injection of placebo in altering the vitreoretinal interface significantly, with the resolution of more vitreomacular tractions and the closure of more macular holes, than that accomplished by placebo treatment of the affected eyes. Therefore, Codenotti $e^{a} \mathrm{al}^{3}$ should wait longer to conclude that 'a single intravitreal APE (autologous plasmin enzyme) injection seems insufficient to induce a complete posterior vitreous detachment in these patients'. In previous studies, there was no statistically significant difference between placebo and microplasmin before 7 days, but it was significant for all comparisons after 7 days, especially as there seemed to be a marked difference during surgery in the adhesion of the posterior hyaloid between autologous plasmin enzyme and placebo treatment in their study. ${ }^{3}$

\section{Conflict of interest}

The authors declare no conflict of interest.

\section{References}

1 Stalmans P, Benz MS, Gandorfer A, Kampik A, Girach A, Pakola $S$ et al. MIVI-TRUST Study Group. Enzymatic 
vitreolysis with ocriplasmin for vitreomacular traction and macular holes. N Engl J Med 2012; 16: 606-615.

2 Tsui I, Pan CK, Rahimy E, Schwartz SD. Ocriplasmin for vitreoretinal diseases. J Biomed Biotechnol. 2012; 2012: 354979.

3 Codenotti M, Maestranzi G, De Benedetto U, Querques G, Della Valle P, Iuliano L et al. Vitreomacular traction syndrome: a comparison of treatment with intravitreal plasmin enzyme $v s$ spontaneous vitreous separation without treatment. Eye 2013; 27: 22-27.

F Matonti ${ }^{1,2}$, JC Courjaret ${ }^{1}$, S Nadeau ${ }^{1}$ and D Denis ${ }^{1}$

${ }^{1}$ Ophthalmology Department, Aix-Marseille

University, Hôpital Nord, Marseille, France

${ }^{2}$ Institut de Neurosciences Cognitives de la

Méditerranée (CNRS 6193), Aix-Marseille University, Marseille, France

E-mail: frederic.matonti@ap-hm.fr

Eye (2013) 27, 777-778; doi:10.1038/eye.2013.62; published online 12 April 2013

Sir, Reply to Matonti et al

We thank Dr Matonti et al ${ }^{1}$ for their interest in and comments on our recent article. ${ }^{2}$ We agree that in our study we should have been waiting longer to definitely assess the effects of autologous plasmin enzyme (APE) in patients with vitreomacular traction syndrome. In fact, although we could not ascertain if a greater time gap could have influenced the rate of posterior vitreous detachment occurrence, as Dr Matonti et al correctly pointed out, we remarked that the single injection appeared as a useful tool in vitreoretinal surgery because of obtaining an easier-to-peel posterior hyaloid. ${ }^{3}$

However, as per our ethical committee-approved protocol (reported in the Methods section), and due to medicolegal reasons, we were allowed to perform just one single intravitreal injection for each study patient, with a 24-h waiting time before vitrectomy. This, together with the impossibility (per protocol) to re-inject patients with APE, should be acknowledged as the limitation of our study.

\section{Conflict of interest}

The authors declare no conflict of interest.

\section{References}

1 Matonti F, Courjaret JC, Nadeau S, Denis D. Comment on 'Vitreomacular traction syndrome: a comparison of treatment with intravitreal plasmin enzyme vs spontaneous vitreous separation without treatment'. Eye 2013; 27(6): 777-778.

2 Codenotti M, Maestranzi G, De Benedetto U, Querques G, Della Valle P, Iuliano L et al. Vitreomacular traction syndrome: a comparison of treatment with intravitreal plasmin enzyme vs spontaneous vitreous separation without treatment. Eye 2013; 27: 22-27.
3 Azzolini C, D'Angelo A, Maestranzi G, Codenotti M, Della Valle P, Prati $\mathrm{M}$ et al. Intrasurgical plasmin enzyme in diabetic macular edema. Am J Ophthalmol 2004; 138: 560-566.

G Querques, M Codenotti, G Maestranzi, U De Benedetto and $\mathrm{F}$ Bandello

Department of Ophthalmology, Vita-Salute University, San Raffaele Scientific Institute, Milan, Italy

E-mail: giuseppe.querques@hotmail.it

Eye (2013) 27, 778; doi:10.1038/eye.2013.64;

published online 12 April 2013

Sir,

Accurate biometry in silicone oil-filled eyes

Kunavisarut et al ${ }^{1}$ should be congratulated on their study 'Accuracy and reliability of IOL master and A-scan immersion biometry in silicone oil-filled eyes'.

This is an important work, given the difficulties that can be encountered in IOL power prediction in these cases. As stated by the authors, it is not always possible to obtain accurate axial length measurements in these eyes preoperatively and the use of data from the fellow eye can be inaccurate.

This paper compared the preoperative axial length measurements for IOL master and immersion ultrasound in oil-filled eyes with the measurement from the IOL master taken after removal of the oil. Accurate axial length measurement is a critical part of biometry in cataract surgery, as an error in this measurement is multiplied by a factor of around 2.5 in the process of intraocular lens power calculation. ${ }^{2}$

In this study, the authors used correlation coefficients to compare the axial length measurements obtained pre and post oil removal. Although the IOL master axial length measurements for silicone oil-filled eyes showed a high correlation with the postoperative axial length measurement (0.966), the postoperative refractive outcomes varied between -2.74 and +2.33 for this relatively small sample of eyes. As stated by Bland and Altman, ${ }^{3}$ the use of correlation coefficients to compare two different measurements of the same thing is problematic since even a very high correlation does not necessarily translate into useful clinical agreement.

The method described by Bland and Altman allows 95\% limits of agreement to be calculated. To achieve this, the differences between the measured values are plotted against their means. This method may have better illustrated why there were clinically significant errors in the refractive outcomes for the IOL master, despite a high correlation between the axial length measurements and no statistically significant difference between the mean measurements taken pre and post oil removal.

\section{Conflict of interest}

The author declares no conflict of interest. 